\title{
On the sum of a prime and the $k$ th power of a prime
}

by

\author{
Claus Bauer (Freiburg)
}

1. Introduction and statement of results. In the last few years a number of authors have investigated nonlinear problems in additive prime number theory for short intervals. Perelli and Pintz [7] and Mikawa [5] have shown independently that in an interval $[x, x+y]$ with $x^{7 / 24+\varepsilon} \leq y \leq x$, all but $\ll_{c} y(\log x)^{-c}$ integers can be represented as the sum of a prime number and a square of a natural number, where $c$ is any positive constant. A similar result was achieved by Perelli and Zaccagnini [8] for the sum of a prime number and the $k$ th power of a natural number for a fixed integer $k \geq 2$. Zhan and Liu [13] have proved the following result: Define

$$
\begin{array}{r}
E_{k}(x)=\mid\left\{n: n \leq x, 2 \mid n, n \neq 1(\bmod p) \forall p>2 \text { with } p-1 \mid k, n \neq p_{1}+p_{2}^{k}\right. \\
\text { for all prime numbers } \left.p_{1}, p_{2}\right\} \mid .
\end{array}
$$

Then

$$
E_{2}(x+y)-E_{2}(x) \ll y(\log x)^{-A}
$$

for $x^{7 / 16+\varepsilon} \leq y \leq x$. We are going to generalize this result for all $k \geq 2$ by proving the following theorem:

Theorem 1. For any $k \geq 2$, any $A>0$ and any $\varepsilon>0$,

$$
E_{k}(x+y)-E_{k}(x) \ll y(\log x)^{-A}
$$

for $x^{\frac{7}{12}\left(1-\frac{1}{2 k}\right)+\varepsilon} \leq y \leq x$, where the $\ll$-constant depends at most on $k, A$ and $\varepsilon$.

Applying a standard argument we will derive this estimate from the following theorem. Let $\Lambda(n), \mu(n)$ and $\phi(n)$ denote the von Mangoldt, the

1991 Mathematics Subject Classification: 11F32, 11F25.

This article forms a part of the author's doctoral dissertation supervised by Professor Dr. D. Wolke from the Department of Mathematics at the University of Freiburg, Germany. During the preparation of this article the author was holding a common scholarship by the Chinese State Education Commission and the German Academic Exchange Service (DAAD). 
Möbius and the Euler function respectively and write $e(\alpha)=e^{2 \pi i \alpha}$. For any fixed integer $k$ and any integer $d \in\{1, k\}$ define

$$
\begin{aligned}
& \sum_{m=1}^{q} * \sum_{\substack{m=1 \\
(m, q)=1}}^{q}, \quad C_{d}(q, a)=\sum_{m=1}^{q} *\left(\frac{m^{d} a}{q}\right), \\
& A(q, n)=\sum_{a=1}^{q} C_{1}(q, a) C_{k}(q, a) e\left(\frac{-a n}{q}\right), \quad \sigma(n, R)=\sum_{q \leq R} \frac{A(q, n)}{\phi^{2}(q)}, \\
& R(n)=\sum_{\begin{array}{c}
n=m_{1}+m_{2}^{k} \\
x-y<m_{1} \leq x \\
y / 2^{k}<m_{2}^{k} \leq\left(2^{k}+1\right) y / 2^{k}
\end{array}} \Lambda\left(m_{1}\right) \Lambda\left(m_{2}\right), \quad P(n)=\sum_{\begin{array}{c}
n=m_{1}+m_{2}^{k} \\
x-y<m_{1} \leq x \\
y / 2^{k}<m_{2}^{k} \leq\left(2^{k}+1\right) y / 2^{k}
\end{array}}
\end{aligned}
$$

We are going to show

Theorem 2. For any fixed $k \geq 2$, any $A>0$ and any $\varepsilon>0$,

$$
\sum_{x<n \leq x+H}|R(n)-\sigma(n, P) P(n)|^{2} \ll H y^{2 / k}(\log x)^{-A}
$$

for $P=(\log x)^{B_{1}}$, where $B_{1}=B_{1}(A)$ is a sufficiently large constant, $x^{7 / 12+\varepsilon} \leq y \leq x$ and $y^{1-1 / 2 k+\varepsilon} \leq H \leq y$. The «-constant depends at most on $k, A$ and $\varepsilon$.

Our results are weaker than Perelli and Zaccagnini's analogous results in [8], who in our notation can choose $H$ in Theorem 2 as small as $\max \left(y^{1-1 / k+\varepsilon}, x^{1 / 2+\varepsilon}\right)$ and therefore obtain an estimate for the corresponding exceptional set for $y$ as small as $\max \left(x^{\frac{7}{12}\left(1-\frac{1}{k}\right)+\varepsilon}, x^{1 / 2+\varepsilon}\right)$. This is due to the fact that we need a mean value estimate for nonlinear trigonometric sums over primes and not just over natural numbers as given by Perelli and Zaccagnini. We can only establish this estimate for a range of $H$ longer than the one in $[8]$.

2. Notation and structure of the proof. Furthermore, we will use the following notation:

$$
\begin{gathered}
D_{1}(\alpha)=\sum_{x-y<m \leq x} \Lambda(m) e(m \alpha), \quad D_{k}(\alpha)=\sum_{y / 2^{k}<m_{2}^{k} \leq\left(2^{k}+1\right) y / 2^{k}} \Lambda(m) e\left(m^{k} \alpha\right), \\
I_{1}(\alpha)=\sum_{x-y<m \leq x} e(m \alpha), \quad I_{k}(\alpha)=\sum_{\substack{y / 2^{k}<m_{2}^{k} \leq\left(2^{k}+1\right) y / 2^{k} \\
m \sim M}} e\left(m^{k} \alpha\right), \\
m \sim M \leq m<2 M .
\end{gathered}
$$

$c$ and $\varepsilon$ denote positive constants which depend at most on $k$ and can take different values on different occasions. By $\|x\|$ we denote the distance from 
$x$ to the nearest integer. We set

$$
L=\log x, \quad Q=H L^{-B_{2}}, \quad P=L^{B_{1}},
$$

where $B_{1}$ and $B_{2}$ will be determined in the sequel. Without further references we shall make use of the relations $\log x \ll \log y \ll \log H$. The major $\operatorname{arcs} M$ and the minor arcs $m$ are defined by

$$
M=\bigcup_{q \leq P} \bigcup_{\substack{a=1 \\(a, q)=1}}^{q}\left[\frac{a}{q}-\frac{1}{Q}, \frac{a}{q}+\frac{1}{Q}\right], \quad m=\left[-\frac{1}{Q}, 1-\frac{1}{Q}\right] \backslash M .
$$

Thus we arrive at

$$
\begin{aligned}
& \sum_{x<n \leq x+H}|R(n)-\sigma(n, P) P(n)|^{2} \\
& =\sum_{x<n \leq x+H}\left|\int_{-1 / Q}^{1-1 / Q} D_{1}(\alpha) D_{k}(\alpha) e(-n \alpha) d \alpha-\sigma(n, P) P(n)\right|^{2} \\
& \quad \ll \sum_{x<n \leq x+H}\left|\int_{M} D_{1}(\alpha) D_{k}(\alpha) e(-n \alpha) d \alpha-\sigma(n, P) P(n)\right|^{2} \\
& \quad+\sum_{x<n \leq x+H}\left|\int_{m} D_{1}(\alpha) D_{k}(\alpha) e(-n \alpha) d \alpha\right|^{2} \\
& =: \sum_{M}+\sum_{m} .
\end{aligned}
$$

3. The minor arcs. In order to estimate the contribution of the integral over the minor arcs, we shall establish Lemma 3.3 below. For this purpose we will first give some results and definitions from [4]. For any positive integers $x, y$ and $r$ with $1 \leq r \leq x, x^{\varepsilon} \leq y \leq x$ and any real number $\alpha=a / q+\theta / q^{2}$, $(a, q)=1,|\theta| \leq 1$, we have:

$$
\begin{gathered}
\sum_{x<n \leq x+y} \tau^{c}(n) \tau^{c}(n+r) \ll y(\log x)^{c}, \\
\sum_{n \leq y} \tau^{c}(n) \min \left(x, \frac{1}{\|n \alpha\|}\right) \\
\ll\left(x y q^{-1 / 2}+x y^{1 / 2}+x^{1 / 2} y+(x y q)^{1 / 2}\right)(\log x y q)^{c}
\end{gathered}
$$

(see (3.3) and (3.4) of [4]).

For any arithmetic function $g(n)$ we define

$$
\begin{aligned}
\nabla\left(g(n) ; v_{1}\right) & =g(n) g\left(n+v_{1}\right), \\
\nabla\left(g(n) ; v_{1}, \ldots, v_{j}\right) & =\nabla\left(\nabla\left(g(n) ; v_{1}, \ldots, v_{j-1}\right) ; v_{j}\right) .
\end{aligned}
$$


Thus

$$
\nabla\left(\left(g_{1} g_{2}\right)(n) ; v_{1}, \ldots, v_{j}\right)=\nabla\left(g_{1}(n) ; v_{1}, \ldots, v_{j}\right) \nabla\left(g_{2}(n) ; v_{1}, \ldots, v_{j}\right)
$$

and for $g(n) \ll G(n)$,

$$
\nabla\left(g(n) ; v_{1}, \ldots, v_{j}\right) \ll \nabla\left(G(n) ; v_{1}, \ldots, v_{j}\right) .
$$

For a polynomial $f(n)$ with real coefficients we set

$$
\begin{aligned}
\Delta\left(f(n) ; v_{1}\right) & =f\left(n+v_{1}\right)-f(n), \\
\Delta\left(f(n) ; v_{1}, \ldots, v_{j}\right) & =\Delta\left(\Delta\left(f(n) ; v_{1}, \ldots, v_{j-1}\right) ; v_{j}\right) .
\end{aligned}
$$

For $f(n)=\beta n^{k}$ and two polynomials $f_{1}(n)$ and $f_{2}(n)$ we thus obtain

$$
\begin{aligned}
& \Delta\left(f(n) ; v_{1}, \ldots, v_{k-1}\right) \\
& =\beta k ! v_{1} \ldots v_{k-1} n+\beta \frac{k !}{2} \sum_{\substack{a_{1}+\ldots+a_{k-1}=k \\
a_{i} \geq 1}} v_{1}^{a_{1}} \ldots v_{k-1}^{a_{k-1}}, \\
& \begin{array}{c}
\Delta\left(f(n) ; v_{1}, \ldots, v_{k}\right)=\beta k ! v_{1} \ldots v_{k}, \\
\Delta\left(\left(f_{1}+f_{2}\right)(n) ; v_{1}, \ldots, v_{k-1}\right) \\
=\Delta\left(f_{1}(n) ; v_{1}, \ldots, v_{k-1}\right)+\Delta\left(f_{2}(n) ; v_{1}, \ldots, v_{k-1}\right) .
\end{array}
\end{aligned}
$$

For positive numbers $x$ and $y$, an arithmetic function $g(n)$ which only takes positive values and a polynomial $f(n)$ with real coefficients we furthermore define

$$
S=\sum_{x<n \leq x+y} g(n) e(f(n)) .
$$

Thus for each integer $j \geq 1$ we have

$$
\begin{aligned}
|S|^{2^{j}} \ll & y^{2^{j}-j-1} \\
& \times \sum_{v_{1}} \ldots \sum_{v_{j}} \sum_{n} \nabla\left(g(n) ; v_{1}, \ldots, v_{j}\right) e\left(\Delta\left(f(n) ; v_{1}, \ldots, v_{j}\right)\right),
\end{aligned}
$$

where the $v_{i}$ run over all integers and for any fixed $v_{1}, \ldots, v_{j}$ the summation over $n$ is restricted by the inequalities

$$
x<n+\sigma(j) \leq x+y,
$$

where $\sigma(j)$ runs over the set

$$
\Sigma(j)=\left\{\sum_{z \in Z} z: Z \text { is any subset of }\left\{v_{1}, \ldots, v_{j}\right\}\right\} .
$$

Finally,

$$
\sum_{v_{1} \ll y} \ldots \sum_{v_{j} \ll y} \sum_{n \sim N} \nabla\left(\tau^{c}(n) \tau^{c}(n+r) ; v_{1}, \ldots, v_{j}\right) \ll y^{j} N(\log y)^{c}
$$

for $N^{\varepsilon} \ll y \ll N$ and $r \ll N$. 
The above statements can all be found in [4], (3.5)-(3.10), Lemmas 3.1 and 3.2 or they follow straight from the definitions.

In the next three lemmas we use $L$ to denote $\log y$ (and not $\log x$ as before).

LEMMA 3.1. Let $a_{m}$ and $b_{m}$ for $m \geq 0$ be real numbers satisfying $a_{m} \ll$ $\tau^{c}(m)$ and $b_{m} \ll \tau^{c}(m)$. Then for every fixed number $k \geq 2$ and any $A>0$ there exists a $B_{3}=B_{3}(A)>0$ such that for $B \geq B_{3}$ the estimate

$$
\int_{y}^{2 y}\left|\sum_{t<m^{k} n^{k} \leq t+H, m \sim M} a_{m} b_{n} e\left(m^{k} n^{k} \alpha\right)\right|^{2} d t \ll H^{2} y^{2 / k-1} L^{-A}
$$

holds for $\alpha=a / q+\theta / q^{2},(a, q)=1,|\theta| \leq 1, L^{B} \leq q \leq H L^{-B}, y^{1-1 / k} \leq$ $H \leq y, L^{B} \leq M \leq 2 H y^{1 / k-1} L^{-B}$. The $\ll$-constant depends at most on $k$ and $A$. The lemma also holds if the summation range of $n$ is shortened.

Proof. Let $K=2^{k-1}$ and $J_{1}$ denote the left-hand side in (3.10). By Cauchy's inequality and (3.1) we thus see

$$
\begin{aligned}
J_{1} \ll & M L^{c} \sum_{m \sim M} \int_{y}^{2 y}\left|\sum_{t<m^{k} n^{k} \leq t+H} b_{n} e\left(m^{k} n^{k} \alpha\right)\right|^{2} d t \\
= & M L^{c} \sum_{m \sim M} \sum_{n_{1}} \sum_{\substack{n_{2}, n_{1} \neq n_{2} \\
y<m^{k} n_{1}^{k} \leq 2 y+H}} b_{n_{1}} b_{n_{2}} e\left(m^{k}\left(n_{1}^{k}-n_{2}^{k}\right) \alpha\right) \int_{T_{1}}^{T_{2}} 1 d t \\
& +O\left(M L^{c} M H \sum_{n \ll y^{1 / k} / M} \tau^{c}(n)\right),
\end{aligned}
$$

where $T_{1}=\max \left(m^{k} n_{1}^{k}-H, m^{k} n_{2}^{k}-H\right)$ and $T_{2}=\min \left(m^{k} n_{1}^{k}, m^{k} n_{2}^{k}\right)$. Set $n_{1}-n_{2}=r, n_{2}=n$ and $g(m, n, r)=H-m^{k} r\left(n^{k-1}+\ldots+(n+r)^{k-1}\right)$. As $\int_{T_{1}}^{T_{2}} 1 d t=0$, if not $m^{k}\left|n_{1}^{k}-n_{2}^{k}\right| \leq H$, we can assume that $|r| \ll$ $H M^{-k} M^{k-1} y^{-(k-1) / k}=H M^{-1} y^{-(k-1) / k}$, and also $r>0$. By $R_{l}(n)$ we denote a polynomial in at least the variable $n$ whose degree relative to $n$ is not greater than $l$. For a sufficiently large $B$, by using (3.1), Hölder's inequality, (3.5) and (3.6) we obtain

$$
\begin{aligned}
& \left|J_{1}\right|^{K / 2} \\
& \ll \mid M L^{c} \sum_{0<r \ll H /\left(M y^{(k-1) / k}\right)} \sum_{m \sim M} \sum_{\begin{array}{c}
y^{1 / k} / M \ll n \ll y^{1 / k} / M \\
m^{k} r\left(n^{k-1}+\ldots+(n+r)^{k-1}\right) \leq H
\end{array}} g(m, n, r) \\
& \quad \times\left. b_{n} b_{n+r} e\left(m^{k} r k n^{k-1} \alpha+m^{k} R_{k-2}(n) \alpha\right)\right|^{K / 2}+H^{K} y^{K(2-k) / 2 k} L^{-K A / 2} \\
& \ll M^{K / 2}\left(H y^{(1-k) / k} M^{-1} M\right)^{K / 2-1}
\end{aligned}
$$




$$
\begin{aligned}
& \times L^{c} \sum_{0<r \ll H /\left(M y^{(k-1) / k}\right)} \sum_{m \sim M} \mid \sum_{\begin{array}{c}
y^{1 / k} / M \ll n \ll y^{1 / k} / M \\
m^{k} r\left(n^{k-1}+\ldots+(n+r)^{k-1}\right) \leq H
\end{array}} g(m, n, r) \\
& \times\left. b_{n} b_{n+r} e\left(m^{k} r k n^{k-1} \alpha+m^{k} R_{k-2}(n) \alpha\right)\right|^{K / 2}+H^{K} y^{K(2-k) / 2 k} L^{-K A / 2} \\
& \ll H^{K / 2-1} M^{K / 2} y^{(1-k)(K / 2-1) / k}\left(\frac{y^{1 / k}}{M}\right)^{K / 2-k+1} L^{c} \\
& \times \sum_{0<r \ll H /\left(M y^{(k-1) / k}\right)} \sum_{m} \sum_{v_{1}} \ldots \sum_{v_{k-2}} \sum_{n} \nabla\left(g(m, n, r) b_{n} b_{n+r} ; v_{1}, \ldots, v_{k-2}\right) \\
& \times e\left(m^{k} r k ! v_{1} \ldots v_{k-2} n \alpha+m^{k} R_{0}(n) \alpha\right)+O\left(H^{K} y^{K(2-k) / 2 k} L^{-K A / 2}\right), \\
& \left|J_{1}\right|^{K^{2} / 2} \\
& \ll H^{K^{2} / 2-K} y^{K^{2}(2-k) / 2 k} M^{K(k-1)}\left(\frac{H}{M y^{(k-1) / k}} \cdot \frac{y^{(k-1) / k}}{M^{k-1}}\right)^{K-1} L^{c} \\
& \times \sum_{0<r \ll H /\left(M y^{(k-1) / k}\right)} \sum_{v_{1}} \ldots \sum_{v_{k-2}} \sum_{n} \mid \sum_{m} \nabla\left(g(m, n, r) ; v_{1}, \ldots, v_{k-2}\right) \\
& \times\left. e\left(m^{k} r k ! v_{1} \ldots v_{k-2} n \alpha+m^{k} R_{0}(n) \alpha\right)\right|^{K} \\
& +H^{K^{2}} y^{K^{2}(2-k) / 2 k} L^{-K^{2} A / 2},
\end{aligned}
$$

where the summations are as stated before. Applying (3.5) and (3.6) to the inner sum over $m$ we obtain

$$
\begin{aligned}
& \left|\sum_{m}\right|^{K} \\
& \ll M^{K-k} \sum_{u_{1}} \ldots \sum_{u_{k-1}} \sum_{m} \nabla\left(\nabla\left(g(m, n, r) ; v_{1}, \ldots, v_{k-2}\right) ; u_{1}, \ldots, u_{k-1}\right) \\
& \quad \times e\left(m n r(k !)^{2} v_{1} \ldots v_{k-2} u_{1} \ldots u_{k-1} \alpha\right. \\
& \left.\quad+n r \frac{(k !)^{2}}{2} v_{1} \ldots v_{k-2}\left(\sum_{a_{1}+\ldots+a_{k-1}=k} u_{1}^{a_{1}}+\ldots+u_{k-1}^{a_{k-1}}\right) \alpha+T(m) \alpha\right),
\end{aligned}
$$

where $\left|u_{1}\right| \ll M, \ldots,\left|u_{k-1}\right| \ll M, m+\sigma^{*}(k-1) \sim M,\left(m+\sigma^{*}(k-1)\right)^{k} r((n+$ 
$\left.\sigma(k-2))^{k-1}+\ldots+(r+n+\sigma(k-2))^{k-1}\right) \leq H$ and $T(m)$ depends on $m$, but not on $n$. Substituting the last estimate in (3.11), using partial summation,

$$
\nabla\left(\nabla\left(g(m, n, r) ; v_{1}, \ldots, v_{k-2}\right) ; u_{1}, \ldots, u_{k-1}\right) \leq H^{K^{2} / 2}
$$

and $\sum_{A<n<B} e(n \alpha) \ll \min (B-A, 1 /\|\alpha\|)$ we find that

$$
\begin{aligned}
& \left|J_{1}\right|^{K^{2} / 2} \\
& \ll H^{K^{2} / 2-K} y^{K^{2}(2-k) / 2 k} M^{K(k-1)}\left(\frac{H}{M^{k}}\right)^{K-1} M^{K-k} L^{c} \\
& \times \sum_{0<r \ll H /\left(M y^{(k-1) / k}\right)} \sum_{v_{1}} \ldots \sum_{v_{k-2}} \sum_{u_{1}} \ldots \\
& \ldots \sum_{u_{k-1}} \sum_{m} \mid \sum_{n} \nabla\left(\nabla\left(g(m, n, r) ; v_{1}, \ldots, v_{k-2}\right) ; u_{1}, \ldots, u_{k-1}\right) \\
& \times e\left(n r \frac{(k !)^{2}}{2} v_{1} \ldots v_{k-2} u_{1} \ldots u_{k-1}\left(2 m+u_{1}+\ldots+u_{k-1}\right) \alpha\right) \\
& +H^{K^{2}} y^{K^{2}(2-k) / 2 k} L^{-K^{2} A / 2} \\
& \ll H^{K^{2}-1} y^{K^{2}(2-k) / 2 k} L^{c} \sum_{0<r \ll H /\left(M y^{(k-1) / k}\right)} \sum_{v_{1}} \ldots \sum_{v_{k-2}} \sum_{u_{1}} \ldots \\
& \cdots \sum_{u_{k-1}} \sum_{m} \min \left(\frac{y^{1 / k}}{M}\right. \\
& \left.\frac{1}{\left\|r \frac{(k !)^{2}}{2} v_{1} \ldots v_{k-2} u_{1} \ldots u_{k-1}\left(2 m+u_{1}+\ldots+u_{k-1}\right) \alpha\right\|}\right) \\
& +H^{K^{2}} y^{K^{2}(2-k) / 2 k},
\end{aligned}
$$

where the summations are as stated before. The expression inside $\|$ \| can only be zero if at least one $u_{i}$ or one $v_{i}$ is 0 . (The expression in brackets is equal to $m+\left(m+u_{1}+\ldots+u_{k-1}\right)$ and so $\neq 0$ because $m+\sigma(k-1) \sim M$.) Thus the contribution of these terms to (3.12) is

$$
\begin{aligned}
& \ll \frac{y^{1 / k}}{M} \cdot \frac{H}{M y^{(k-1) / k}}\left(\frac{y^{(k-3) / k}}{M^{k-3}} M^{k-1}+\frac{y^{(k-2) / k}}{M^{k-2}} M^{k-2}\right) M \\
& \ll H L^{-K^{2} A / 2-c} .
\end{aligned}
$$

The number of terms which satisfy

$$
0 \neq n=r \frac{(k !)^{2}}{2} v_{1} \ldots v_{k-2} u_{1} \ldots u_{k-1}\left(2 m+u_{1}+\ldots+u_{k-1}\right)
$$


is $\leq \tau^{2 k-2}(n)$, because $r, u_{i}$ and $v_{j}$ respectively divide $n$ and for fixed $r, u_{i}$ and $v_{j}$ there is at most one possible choice for $m$. We can derive from

$$
n \ll \frac{H}{M y^{(k-1) / k}}\left(\frac{y^{1 / k}}{M}\right)^{k-2} M^{k-1} M=H M y^{-1 / k}
$$

and (3.2) that these terms do not contribute to (3.12) more than

$$
\begin{aligned}
& \ll \sum_{0<n \ll H M y^{-1 / k}} \tau^{c}(n) \min \left(\frac{y^{1 / k}}{M}, \frac{1}{\|n \alpha\|}\right) \\
& \ll H L^{c}\left(q^{-1 / 2}+M^{1 / 2} y^{-1 / 2 k}+H^{-1 / 2} M^{-1 / 2} y^{1 / 2 k}+H^{-1 / 2} q^{1 / 2}\right) \\
& \ll H L^{-K^{2} A / 2-c},
\end{aligned}
$$

if $B$ is chosen arbitrarily large. Now the lemma follows from the last estimate, (3.12) and (3.13).

Lemma 3.2. Let $a_{m}$ denote real numbers which satisfy $a_{m} \ll \tau^{c}(m)$. For any integer $k \geq 2$ and any $A>0$ there exists a $B_{4}=B_{4}(A)>0$ such that for $B \geq B_{4}$ the estimate

$$
\int_{y}^{2 y}\left|\sum_{t<m^{k} n^{k} \leq t+H, m \sim M} a_{m} e\left(m^{k} n^{k} \alpha\right)\right|^{2} d t \ll H^{2} y^{2 / k-1} L^{-A}
$$

holds for $\alpha=a / q+\theta / q^{2},(a, q)=1,|\theta| \leq 1, L^{B} \leq q \leq H L^{-B}, y^{1-1 / k} L^{B} \leq$ $H \leq y$ and $M^{2^{k-2}} \leq y^{1 / 2 k} L^{-B}$. The $\ll$-constant depends at most on $k$ and $A$.

REMARK. Under the conditions of Lemma 3.2,

$$
\int_{y}^{2 y}\left|\sum_{t<m^{k} n^{k} \leq t+H, m \sim M}(\log n) a_{m} e\left(m^{k} n^{k} \alpha\right)\right|^{2} d t \ll H^{2} y^{2 / k-1} L^{-A} .
$$

The lemma and the remark also apply if the summation range of $n$ is shortened.

Pr o of (of Lemma 3.2). Let $J_{2}$ denote the left-hand side in (3.14). Following the same lines as in the proof of Lemma 3.1 we arrive at

$$
\begin{aligned}
J_{2}= & \int_{y}^{2 y} \sum_{m_{1} \sim M} \sum_{m_{2} \sim M} \sum_{n_{1}} \sum_{\substack{n_{2} \\
m_{1} n_{1} \neq m_{2} n_{2} \\
t<\left(m_{i} n_{i}\right)^{k} \leq t+H}} a_{m_{1}} a_{m_{2}} \\
& \times e\left(\left(\left(m_{2} n_{2}\right)^{k}-\left(m_{1} n_{1}\right)^{k}\right) \alpha\right) d t+O\left(H \sum_{n \ll y^{1 / k}} \tau^{c}(n)\right)
\end{aligned}
$$




$$
\begin{aligned}
= & \sum_{m_{1} \sim M} \sum_{\substack{m_{2} \sim M \\
n_{1}}} a_{m_{1}} a_{m_{2}} \\
& \times \sum_{\substack{n_{2} \\
0<\left|\left(m_{2} n_{2}\right)^{k}-\left(m_{1} n_{1}\right)^{k}\right| \leq H \\
y<\left(m_{i} n_{i}\right)^{k} \leq 2 y+H}}\left(H-\left|\left(m_{2} n_{2}\right)^{k}-\left(m_{1} n_{1}\right)^{k}\right|\right) \\
& \times e\left(\left(m_{2} n_{2}-m_{1} n_{1}\right)\left(\left(m_{1} n_{1}\right)^{k-1}+\ldots+\left(m_{2} n_{2}\right)^{k-1}\right) \alpha\right) \\
& +O\left(H y^{1 / k} L^{c}\right) .
\end{aligned}
$$

Let $r=m_{2} n_{2}-m_{1} n_{1}, \delta=\left(m_{1}, m_{2}\right), m_{1}=\delta m_{1}^{*}, m_{2}=\delta m_{2}^{*}, n=n_{1}$ and without loss of generality assume $r>0$. Then $\left(m_{1}^{*}, m_{2}^{*}\right)=1$ and $\delta \mid r$. Writing $r=\delta r^{*}$ and noting that

$$
r^{*} \delta=\frac{\left(m_{2}^{*} n_{2}\right)^{k}-\left(m_{1}^{*} n_{1}\right)^{k}}{\left(m_{1}^{*} n_{1}\right)^{k-1}+\ldots+\left(m_{2}^{*} n_{2}\right)^{k-1}} \delta \ll H y^{(1-k) / k},
$$

we arrive at

$$
\begin{aligned}
J_{2}= & \sum_{\delta \ll M} \sum_{0<r^{*} \ll H y^{1 / k-1} \delta^{-1}} \sum_{m_{1}^{*} \sim M \delta^{-1}} \sum_{\substack{m_{2}^{*} \sim M \delta^{-1} \\
\left(m_{1}^{*}, m_{2}^{*}\right)=1}} a_{m_{1}^{*} \delta} a_{m_{2}^{*} \delta} \\
& \times \sum_{n}\left(H-k \delta^{k} r^{*}\left(m_{1}^{*} n\right)^{k-1}+P_{k-2}\left(m_{1}^{*} n\right)\right) \\
& \times e\left(k \delta^{k} r^{*}\left(m_{1}^{*} n\right)^{k-1} \alpha+P_{k-2}\left(m_{1}^{*} n\right) \alpha\right)+O\left(H y^{1 / k} L^{c}\right),
\end{aligned}
$$

where $P_{k-2}\left(m_{1}^{*} n\right)$ is a polynomial in $m_{1}^{*} n, \delta$ and $r^{*}$ with only positive coefficients, and its degree relative to $m_{1}^{*} n$ is not greater than $k-2$. The summation over $n$ is given by

$$
\begin{gathered}
m_{1}^{*} \delta n \equiv-r^{*} \delta\left(\bmod m_{2}^{*} \delta\right), \quad 0<k \delta^{k} r^{*}\left(m_{1}^{*} n\right)^{k-1}+P_{k-2}\left(m_{1}^{*} n\right) \leq H, \\
y<\left(m_{1}^{*} \delta n+r^{*} \delta\right)^{k} \leq 2 y+H, \quad y<\left(m_{1}^{*} \delta n\right)^{k} \leq 2 y+H .
\end{gathered}
$$

Using (3.1) we see that the terms with $\delta>L^{D}$ do not contribute more than

$$
\begin{aligned}
\sum_{L^{D}<\delta \ll M} \ll & H \sum_{L^{D}<\delta} \tau^{c}(\delta) \sum_{0<r^{*} \ll H y^{1 / k-1} \delta^{-1}} \sum_{m_{1}^{*} \sim M \delta^{-1}} \sum_{m_{2}^{*} \sim M \delta^{-1}} \tau^{c}\left(m_{1}^{*}\right) \tau^{c}\left(m_{2}^{*}\right) \\
& \times \sum_{\left(m_{1}^{*} \delta n\right)^{k} \ll y} \sum_{\substack{n_{2} \\
r^{*}=m_{2}^{*} n_{2}-m_{1}^{*} n}} 1 \\
\ll & H \sum_{L^{D}<\delta} \tau^{c}(\delta) \sum_{0<r^{*} \ll H y^{1 / k-1} \delta^{-1}} \sum_{n \ll y^{1 / k} \delta^{-1}} \tau^{c}(n) \tau^{c}\left(n+r^{*}\right) \\
\ll & H^{2} y^{2 / k-1} \sum_{L^{D}<\delta} \frac{\tau^{c}(\delta)}{\delta^{2}} \ll H^{2} y^{2 / k-1} L^{-A},
\end{aligned}
$$


if $D$ is sufficiently large. So we can concentrate on the case $\delta<L^{D}$. Without loss of generality we assume $\delta=1$ since in the other cases the proof does not change fundamentally. As a consequence we suppose the $m_{1}$ and $m_{2}$ to be relatively prime and write $n=T+v m_{2}$ with $v \geq 0,0 \leq T \ll M, T \equiv-\bar{m}_{1} r$ $\left(\bmod m_{2}\right)$ and $m_{1} \bar{m}_{1} \equiv 1\left(\bmod m_{2}\right)$. Then one can see that it is enough to estimate the following expression which we denote by $J_{2}$ again:

$$
\begin{aligned}
J_{2}= & \sum_{0<r \ll H y^{1 / k-1}} \sum_{m_{1} \sim M} \sum_{m_{2} \sim M} a_{m_{1}} a_{m_{2}} \\
& \times \sum_{v}\left(H-k r\left(m_{1} m_{2} v\right)^{k-1}+P_{k-2}\left(m_{1} m_{2} v\right)\right) \\
& \times e\left(k r\left(m_{1} m_{2} v\right)^{k-1} \alpha+P_{k-2}\left(m_{1} m_{2} v\right) \alpha\right)+O\left(H^{2} y^{2 / k-1} L^{-A}\right),
\end{aligned}
$$

where $v$ runs over

$$
\begin{gathered}
0<k r\left(m_{1} m_{2} v+m_{1} T\right)^{k-1}+P_{k-2}\left(m_{1} m_{2} v+m_{1} T\right) \leq H, \\
y \ll\left(m_{1} m_{2} v+m_{1} T+r\right)^{k} \ll y, \quad y \ll\left(m_{1} m_{2} v+m_{1} T\right)^{k} \ll y .
\end{gathered}
$$

So the maximal range of summation over $v$ is given by

$$
0<r\left(m_{1} m_{2} v\right)^{k-1} \leq H, \quad y \ll\left(m_{1} m_{2} v\right)^{k} \ll y .
$$

In the sequel we still assume the $m_{i}$ and $u_{j}$ to be pairwise coprime. By induction we will show that for $1 \leq j \leq k-1, J=2^{j-1}$ and a sufficiently large $B$ the following holds:

$$
\begin{aligned}
\left|J_{2}\right|^{J} \ll & H^{J-1} y^{1-J}\left(y^{1 / k}\right)^{2 J-j-1} L^{c} \sum_{0<r_{1} \ll H y^{1 / k-1}} \ldots \\
& \ldots \sum_{0<r_{j} \ll H y^{j / k-1} /\left(r_{1} \ldots r_{j-1}\right)} \sum_{m_{1} \sim M} \ldots \sum_{m_{2 J} \sim M} a_{m_{1}} \ldots a_{m_{2 J}} \\
& \times \sum_{n} g\left(r_{1}, \ldots, r_{j}, m_{1}, \ldots, m_{2 J}, m_{1} \ldots m_{2 J} n\right) \\
& \times e\left(k \ldots(k-j+1) r_{1} \ldots r_{j}\left(m_{1} \ldots m_{2 J} n\right)^{k-j} \alpha\right. \\
& \left.+P_{k-j-1}\left(m_{1} \ldots m_{2 J} n\right) \alpha\right)+O\left(H^{2 J} y^{-J} y^{2 J / k} L^{-J A}\right),
\end{aligned}
$$

where the maximal range of summation over $n$ is given by

$$
0 \leq r_{1} \ldots r_{j}\left(m_{1} \ldots m_{2 J} n\right)^{k-j} \ll H, \quad y \ll\left(m_{1} \ldots m_{2 J} n\right)^{k} \ll y,
$$

and $g\left(r_{1}, \ldots, r_{j}, m_{1}, \ldots, m_{2 J}, m_{1} \ldots m_{2 J} n\right) \ll H^{J}$ is a polynomial in the given variables.

For $j=1$, (3.18) follows from (3.16) and (3.17). Suppose that (3.18) holds for a $j$ with $1 \leq j \leq k-2$. By using Cauchy's inequality we get 


$$
\begin{aligned}
& \left|J_{2}\right|^{2 J} \\
\ll & H^{2 J-2} y^{2-2 J}\left(y^{1 / k}\right)^{4 J-2 j-2} L^{c} H y^{j / k-1} \\
& \times \sum_{0<r_{1} \ll H y^{1 / k-1}} \ldots \sum_{0<r_{j} \ll H y^{j / k-1} /\left(r_{1} \ldots r_{j-1}\right)} \sum_{m_{1} \sim M} \ldots \\
& \ldots \sum_{m_{2 J} \sim M} \sum_{u_{1} \sim M} \ldots \sum_{u_{2 J} \sim M} a_{m_{1}} \ldots a_{m_{2 J}} a_{u_{1}} \ldots a_{u_{2 J}} \\
& \times \sum_{n} \sum_{u} g\left(r_{1}, \ldots, r_{j}, m_{1}, \ldots, m_{2 J}, m_{1} \ldots m_{2 J} n\right) \\
& \times g\left(r_{1}, \ldots, r_{j}, u_{1}, \ldots, u_{2 J}, u_{1} \ldots u_{2 J} u\right) e\left(k \ldots(k-j+1) r_{1} \ldots r_{j}\right. \\
& \times\left(\left(u_{1} \ldots u_{2 J} u\right)^{k-j} \alpha-\left(m_{1} \ldots m_{2 J} n\right)^{k-j} \alpha\right)+P_{k-j-1}\left(u_{1} \ldots u_{2 J} u\right) \alpha \\
& \left.-P_{k-j-1}\left(m_{1} \ldots m_{2 J} n\right) \alpha\right)+O\left(H^{4 J} y^{-2 J}\left(y^{1 / k}\right)^{4 J} L^{-2 J A}\right),
\end{aligned}
$$

where the summations over $n$ and $u$ are both given by (3.19). Setting $r_{j+1}=$ $u_{1} \ldots u_{2 J} u-m_{1} \ldots m_{2 J} n$, we obtain

$$
\begin{aligned}
& \left(u_{1} \ldots u_{2 J} u\right)^{k-j}-\left(m_{1} \ldots m_{2 J} n\right)^{k-j} \\
& \quad=r_{j+1}(k-j)\left(m_{1} \ldots m_{2 J} n\right)^{k-j-1}+P_{k-j-2}\left(m_{1} \ldots m_{2 J} n\right),
\end{aligned}
$$

where $P_{k-j-2}$ is a polynomial at least in $m_{1} \ldots m_{2 J} n$ and with degree $\leq$ $k-j-2$ with respect to this variable. By employing the definition of $r_{j+1}$ and (3.19) we also have

$$
\begin{aligned}
r_{j+1} & =\frac{\left(u_{1} \ldots u_{2 J} u\right)^{k-j}-\left(m_{1} \ldots m_{2 J} n\right)^{k-j}}{\left(m_{1} \ldots m_{2 J} n\right)^{k-j-1}+\ldots+\left(u_{1} \ldots u_{2 J} u\right)^{k-j-1}} \\
& \ll \frac{H}{r_{1} \ldots r_{j}} y^{(j+1) / k-1} .
\end{aligned}
$$

We shall assume without loss of generality that $r_{j+1} \geq 0$. Keeping in mind that the $m_{1} \ldots m_{2 J}$ and $u_{1} \ldots u_{2 J}$ were supposed to be coprime we write

$$
n=S+g u_{1} \ldots u_{2 J}
$$

where $m_{1} \ldots m_{2 J} S \equiv-r_{j+1}\left(\bmod u_{1} \ldots u_{2 J}\right), 0 \leq S \ll M^{2 J}$ and $g \geq 0$. From (3.19) and $\left(m_{1} \ldots m_{2 J} S\right)^{k} \ll M^{4 \times 2^{k-3} k} \ll y L^{-2 B k}$ we can derive

$$
y \ll\left(m_{1} \ldots m_{2 J} u_{1} \ldots u_{2 J} g\right)^{k} \ll y .
$$

From (3.19) and (3.23) we further conclude that

$$
0 \leq r_{1} \ldots r_{j+1}\left(m_{1} \ldots m_{2 J} u_{1} \ldots u_{2 J} g\right)^{k-j-1} \ll H .
$$

Taking into account (3.23) we can write 


$$
\begin{aligned}
& g\left(r_{1}, \ldots, r_{j}, m_{1}, \ldots, m_{2 J}, m_{1} \ldots m_{2 J} n\right) \\
& \quad \times g\left(r_{1}, \ldots, r_{j}, u_{1}, \ldots, u_{2 J}, m_{1} \ldots m_{2 J} n+r_{j+1}\right) \\
& \quad:=g\left(r_{1}, \ldots, r_{j+1}, m_{1}, \ldots, m_{2 J}, u_{1}, \ldots, u_{2 J}, m_{1} \ldots m_{2 J} u_{1} \ldots u_{2 J} g\right),
\end{aligned}
$$

and so from (3.20) to (3.25) we obtain

$$
\begin{aligned}
\left|J_{2}\right|^{2 J} \ll & H^{2 J-1} y^{1-2 J}\left(y^{1 / k}\right)^{4 J-j-2} L^{c} \\
& \times \sum_{0<r_{1} \ll H y^{1 / k-1}} \ldots \sum_{0 \leq r_{j+1} \ll H y^{(j+1) / k-1} /\left(r_{1} \ldots r_{j-1} r_{j}\right)} \\
& \times \sum_{m_{1} \sim M} \ldots \sum_{m_{2 J} \sim M} \sum_{u_{1} \sim M} \ldots \sum_{u_{2 J} \sim M} a_{m_{1}} \ldots a_{m_{2 J}} a_{u_{1}} \ldots a_{u_{2 J}} \\
& \times \sum_{g} g\left(r_{1}, \ldots, r_{j+1}, m_{1}, \ldots, m_{2 J},\right. \\
& \left.\quad u_{1}, \ldots, u_{2 J}, m_{1} \ldots m_{2 J} u_{1} \ldots u_{2 J} g\right) \\
& \times e\left(k \ldots(k-j) r_{1} \ldots r_{j} r_{j+1}\left(m_{1} \ldots m_{2 J} u_{1} \ldots u_{2 J} g\right)^{k-j-1}\right. \\
& \left.+P_{k-j-2}\left(m_{1} \ldots m_{2 J} u_{1} \ldots u_{2 J} g\right) \alpha\right) \\
& +O\left(H^{4 J} y^{-2 J}\left(y^{1 / k}\right)^{4 J} L^{-2 J A}\right),
\end{aligned}
$$

where the summation ranges are given by (3.24) and (3.25). Using (3.1) and (3.19) it follows that in (3.20) and therefore also in (3.26) the contribution of the terms with $r_{j+1}=0$ is

$$
\begin{aligned}
& \ll H^{4 J-1} y^{1-2 J}\left(y^{1 / k}\right)^{4 J-j-2} L^{c} \\
& \times \sum_{0<r_{1} \ll H y^{1 / k-1}} \ldots \sum_{0<r_{j} \ll H y^{j / k-1} /\left(r_{1} \ldots r_{j-1}\right)}\left(\frac{H}{r_{1} \ldots r_{j}}\right)^{1 /(k-j)} \\
& \ll H^{4 J} y^{-2 J}\left(y^{1 / k}\right)^{4 J-1} L^{c} \\
& \times \sum_{0<r_{1} \ll H y^{1 / k-1}} \cdots \sum_{0<r_{j-1} \ll H y^{(j-1) / k-1} /\left(r_{1} \ldots r_{j-2}\right)} \frac{1}{r_{1} \ldots r_{j-1}} \\
& \ll H^{4 J} y^{-2 J}\left(y^{1 / k}\right)^{4 J} L^{c} y^{-1 / k} .
\end{aligned}
$$

Now (3.18) follows from (3.26) in the case of $j+1$ if we rename the $u_{1}, \ldots, u_{2 J}$ as $m_{2 J+1}, \ldots, m_{4 J}$. Choosing $j=k-1$ in (3.18) and setting $K=2^{k-2}$, we derive the following result from (3.19) and by using partial summation:

$$
\begin{aligned}
\left|J_{2}\right|^{K} \ll & H^{2 K-1} y^{-K}\left(y^{1 / k}\right)^{2 K} L^{c} \\
& \times \sum_{0<r_{1} \ll H y^{1 / k}} \ldots \sum_{0<r_{k-1} \ll H y^{-1 / k} /\left(r_{1} \ldots r_{k-2}\right)} \sum_{m_{1} \sim M} \ldots \\
& \ldots \sum_{m_{2 K} \sim M} \tau^{c}\left(m_{1} \ldots m_{2 K}\right)
\end{aligned}
$$




$$
\begin{aligned}
& \times \min \left(\frac{H}{r_{1} \ldots r_{k-1} m_{1} \ldots m_{2 K}}, \frac{1}{\left\|k ! r_{1} \ldots r_{k-1} m_{1} \ldots m_{2 K} \alpha\right\|}\right) \\
& +H^{2 K} y^{-K}\left(y^{1 / k}\right)^{2 K} L^{-K A} \\
\ll & H^{2 K-1} y^{-K}\left(y^{1 / k}\right)^{2 K} L^{c} \\
& \times \max _{N \ll H M^{2 K} y^{-1 / k} L^{c}} \sum_{n \sim N} \tau^{c}(n) \min \left(\frac{H}{N}, \frac{1}{\|n \alpha\|}\right) \\
& +H^{2 K} y^{-K}\left(y^{1 / k}\right)^{2 K} L^{-K A},
\end{aligned}
$$

because $r_{1} \ldots r_{k-1} m_{1} \ldots m_{2 K} \ll H M^{2 K} y^{-1 / k} L^{c}$. For $N \geq L^{D_{1}}$ we find

$$
\begin{aligned}
\sum_{n \sim N} \tau^{c}(n) \min & \left(\frac{H}{N}, \frac{1}{\|n \alpha\|}\right) \\
& \ll\left(H q^{-1 / 2}+(H N)^{1 / 2}+H N^{-1 / 2}+(H q)^{1 / 2}\right) L^{c} \\
& \ll\left(H q^{-1 / 2}+H M^{K} y^{-1 / 2 k}+H L^{-D_{1} / 2}+(H q)^{1 / 2}\right) L^{c} \\
& \ll H L^{-K A-c}
\end{aligned}
$$

by applying (3.2) for sufficiently large $B$ and $D_{1}$. For $N \leq L^{D_{1}}$ and $D_{1}$ fixed according to the preceding discussion, we obtain the following for a sufficiently large $B$ :

$$
\begin{aligned}
\sum_{n \sim N} \tau^{c}(n) \min \left(\frac{H}{N}, \frac{1}{\|n \alpha\|}\right) & \ll L \sum_{n \ll L^{D_{1}}} \frac{1}{\|n \alpha\|} \\
& \ll L q \sum_{n \leq L^{D_{1}}} 1 \ll H L^{-K A-c} .
\end{aligned}
$$

The lemma now follows from (3.27)-(3.29).

From Lemmas 3.1 and 3.2 we derive

Lemma 3.3. Let $\alpha=a / q+\theta / q^{2},(a, q)=1$ and $|\theta| \leq 1$. For every fixed $k \geq 2$ and every $A>0$ there exists a $B_{5}=B_{5}(A)>0$ such that for $B \geq B_{5}$,

$$
\int_{y}^{2 y}\left|\sum_{t<m^{k} \leq t+H} \Lambda(m) e\left(m^{k} \alpha\right)\right|^{2} d t \ll H^{2} y^{2 / k-1} L^{-A}
$$

for $L^{B} \leq q \leq H L^{-B}$ and $y^{1-1 / 2 k} L^{B} \leq H \leq y$, where the $\ll$-constant depends at most on $k$ and $A$.

Proof. Set

$$
M(s)=\sum_{n \leq X} \mu(n) n^{-s}, \quad X=2 y^{1 / 2 k}, \operatorname{Re}(s)>1 .
$$


We conclude from Heath-Brown's identity (see [2]) in the form

$$
-\frac{\zeta^{\prime}(s)}{\zeta(s)}=\zeta(s) \zeta^{\prime}(s) M^{2}(s)-2 \zeta^{\prime}(s) M(s)-\frac{\zeta^{\prime}(s)}{\zeta(s)}(1-\zeta(s) M(s))^{2},
$$

that $\sum_{t<m^{k} \leq t+H} \Lambda(m) e\left(m^{k} \alpha\right)$ can be written as $O\left(L^{c}\right)$ sums of the form

$$
\sum=w \sum_{\substack{t<\left(m_{1} \ldots m_{4}\right)^{k} \leq t+H \\ m_{i} \sim M_{i}}} a_{1}\left(m_{1}\right) \ldots a_{4}\left(m_{4}\right) e\left(\left(m_{1} \ldots m_{4}\right)^{k} \alpha\right),
$$

where $|w| \in\{1,2\}, a_{1}\left(m_{1}\right)=\log m_{1}, a_{2}\left(m_{2}\right)=1, a_{3}\left(m_{3}\right)=\mu\left(m_{3}\right)$, $a_{4}\left(m_{4}\right)=\mu\left(m_{4}\right), y \leq\left(M_{1} \ldots M_{4}\right)^{k} \leq 3 y, M_{3} \leq 2 y^{1 / 2 k}, M_{4} \leq 2 y^{1 / 2 k}$. (Some $M_{i}$ may be 1.)

Applying Cauchy's inequality it is obviously enough to show that any integral $\int_{y}^{2 y}\left|\sum\right|^{2} d t$, where $\sum$ is of the type in (3.30), can be estimated sufficiently well.

We distinguish between two cases:

(a) If there exists an $1 \leq j \leq 4$ with $x^{\varepsilon} \leq M_{j} \leq 2 y^{1 / 2 k}$, we can define $a_{1}^{*}\left(m_{1}\right)$ by $a_{1}^{*}\left(m_{1}\right) \log (2 y+H)=a_{1}\left(m_{1}\right)$ and replace $a_{1}\left(m_{1}\right)$ by $a_{1}^{*}\left(m_{1}\right)$ in (3.30). Then by applying the assumption of the lemma and Lemma 3.1 for $M=M_{j}$ we obtain

$$
\int_{y}^{2 y}\left|\sum\right|^{2} d t \ll H^{2} y^{2 / k-1} L^{-A-c} .
$$

(b) If $M_{i}$ satisfies $M_{i}<x^{\varepsilon}$ or $M_{i}>2 y^{1 / 2 k}$ for all $1 \leq i \leq 4$ there exists exactly one $j$ with $M_{j}>2 y^{1 / 2 k}$. We know that in this case $j \leq 2$. For $j=2$ we apply Lemma 3.2 to $M=\prod_{i \neq 2} M_{i} \leq x^{\varepsilon}$. If $j=1$ we apply the remark to Lemma 3.2.

In the sequel we use $L$ again to denote $\log x$. We can now proceed to estimate the sum $\sum_{m}$ in (2.1). Arguing as in Section 3 of [6] we find

$$
\begin{aligned}
\sum_{m} & =\int_{m} D_{1}(\alpha) D_{k}(\alpha) \int_{m} \overline{D_{1}(\beta) D_{k}(\beta)} K(\alpha-\beta) d \beta d \alpha \\
& \ll \int_{m}\left|D_{1}(\alpha) D_{k}(\alpha)\right| \int_{m}\left|D_{1}(\beta) D_{k}(\beta)\right| \min \left(H, \frac{1}{\|\alpha-\beta\|}\right) d \beta d \alpha,
\end{aligned}
$$

where $K(\eta)=\sum_{x<n \leq x+H} e(\eta n)$. Splitting the unit interval in $H$ adjacent, disjoint intervals $H_{i}$ of length $H^{-1}$, we obtain

$$
\begin{aligned}
\sum_{m} \ll & \sum_{1 \leq i \leq H} \sum_{1 \leq j \leq H} \frac{H}{1+|i-j|} \\
& \times \int_{m \cap H_{i}}\left|D_{1}(\alpha) D_{k}(\alpha)\right| \int_{m \cap H_{j}}\left|D_{1}(\beta) D_{k}(\beta)\right| d \alpha d \beta
\end{aligned}
$$




$$
\begin{aligned}
& \ll H \sum_{1 \leq i \leq H}\left(\int_{m \cap H_{i}}\left|D_{1}(\alpha) D_{k}(\alpha)\right| d \alpha\right)^{2} \sum_{1 \leq j \leq H} \frac{1}{1+|i-j|} \\
& \ll H L \sum_{1 \leq i \leq H}\left(\int_{m \cap H_{i}}\left|D_{1}(\alpha)\right|^{2} d \alpha\right)\left(\int_{m \cap H_{i}}\left|D_{k}(\alpha)\right|^{2} d \alpha\right) \\
& \ll H y L^{3} \max _{1 \leq i \leq H} \int_{m \cap H_{i}}\left|D_{k}(\alpha)\right|^{2} d \alpha .
\end{aligned}
$$

If for $1 \leq i \leq H$ we choose a fixed $\alpha \in m \cap H_{i} \subset[\alpha-1 / H, \alpha+1 / H]$, we obtain

$$
\sum_{m} \ll H y^{2 / k} L^{-A}
$$

from (3.31), provided we can show that

$$
\int_{-1 / H}^{1 / H}\left|D_{k}(\alpha+\gamma)\right|^{2} d \gamma \ll y^{2 / k-1} L^{-A-3}
$$

uniformly for all $\alpha \in m$. Applying Gallagher's lemma (see Lemma 1 of [1]) we find

$$
\begin{aligned}
& \int_{-1 / H}^{1 / H}\left|D_{k}(\alpha+\gamma)\right|^{2} d \gamma \\
& \ll H^{-2} \int_{y / 2^{k}-H / 2}^{y / 2^{k}}\left|\sum_{y / 2^{k}<m^{k} \leq t+H / 2} \Lambda(m) e\left(m^{k} \alpha\right)\right|^{2} d t \\
& +\left.H^{-2} \int_{y / 2^{k}} \sum_{t<m^{k} \leq t+H / 2} \Lambda(m) e\left(m^{k} \alpha\right)\right|^{2} d t \\
& \quad+H^{-2} \int_{\left(2^{k}+1\right) y / 2^{k}}\left|\sum_{t<m^{k} \leq\left(2^{k}+1\right) y / 2^{k}} \Lambda(m) e\left(m^{k} \alpha\right)\right|^{2} d t \\
& =: J_{1}+J_{2}+J_{3} .
\end{aligned}
$$

Because of the definition of the minor arcs we can apply Lemma 3.3 to estimate $J_{2}$ by the right side of (3.33). If $H \leq y L^{-A-5}$ a trivial estimate will give

$$
J_{1} \ll H^{-2} H\left(H y^{1 / k-1} L\right)^{2}=H y^{2 / k-2} L^{2} \leq y^{2 / k-1} L^{-A-3} .
$$

Otherwise we use Vinogradov's estimate (Hua [3], Lemma 2) which says 
that for any $\lambda_{0}>0$ the estimate

$$
\sum_{n \leq x} e\left(\alpha p^{k}\right) \ll x L^{-\lambda_{0}},
$$

holds for $\alpha=a / q+\theta / q^{2},(a, q)=1,|\theta| \leq 1, L^{\lambda} \leq q \leq x L^{-\lambda}$ and $\lambda \geq c=$ $c\left(\lambda_{0}\right)$. Applying this to $J_{1}$ we obtain for any sufficiently large $B_{1}$ and $B_{2}$ and $H>y L^{-A-5}$ the following:

$$
J_{1} \ll H^{-2} H y^{2 / k} L^{-2 A-8}<y^{2 / k-1} L^{-A-3} .
$$

Treating $J_{3}$ in the same way and summing up the estimates for the $J_{i}$, we obtain (3.33) and thus (3.32).

4. The major arcs. We will need the following lemma:

Lemma 4.1. For any constants $c>0$ and $A>0$,

$$
\sum_{\chi(\bmod q)} \int_{y}^{2 y}\left|\sum_{t<p \leq t+\theta t}^{\prime}(\log p) \chi(p)\right|^{2} d t \ll_{A, \varepsilon, c} \theta^{2} y^{3} L^{-A}
$$

for $q \leq L^{c}$ and $y^{1 / 6+\varepsilon} \leq y \theta \leq y$, where $\sum^{\prime}$ indicates that if $\chi$ is the principal character, then $\sum_{t<p \leq t+\theta t} \log p$ is replaced by $\sum_{t<p \leq t+\theta t} \log p-\theta t$.

Pr o of. The lemma is a generalization of Selberg's inequality. The proof goes along the same lines as the proofs of Lemmas 5 and 6 in [9].

REMARK. The result is also true if $\theta$ is replaced by $\theta c(t)$, where $c(t)$ is a positive function of $t$ which satisfies $1 \ll c(t) \ll 1$ in the integration interval.

In the sequel we fix $B_{1}$ and choose $B_{2}$ sufficiently large according to the discussion in Section 3. From (2.1) we obtain

$$
\begin{aligned}
\sum_{M} \ll & \sum_{x<n \leq x+H} \mid \sum_{q \leq P} \sum_{a=1}^{q} e\left(-\frac{a}{q} n\right) \\
& \times\left.\int_{-1 / Q}^{1 / Q} D_{1}\left(\frac{a}{q}+\gamma\right)\left(D_{k}\left(\frac{a}{q}+\gamma\right)-\frac{C_{k}(q, a)}{\phi(q)} I_{k}(\gamma)\right) e(-\gamma n) d \gamma\right|^{2} \\
& +\sum_{x<n \leq x+H} \mid \sum_{q \leq P} \sum_{a=1}^{q} \frac{C_{k}(q, a)}{\phi(q)} e\left(-\frac{a}{q} n\right) \\
& \times\left.\int_{-1 / Q}^{1 / Q}\left(D_{1}\left(\frac{a}{q}+\gamma\right)-I_{1}(\gamma) \frac{\mu(q)}{\phi(q)}\right) I_{k}(\gamma) e(-\gamma n) d \gamma\right|^{2} \\
& +\sum_{x<n \leq x+H} \mid \sum_{q \leq P} \sum_{a=1}^{q} \frac{\mu(q) C_{k}(q, a)}{\phi^{2}(q)} e\left(-\frac{a n}{q}\right)
\end{aligned}
$$




$$
\begin{aligned}
& \times\left.\int_{1 / Q}^{1 / 2} I_{1}(\gamma) I_{k}(\gamma) e(-n \gamma) d \gamma\right|^{2} \\
= & \sum_{x<n \leq x+H}\left(\left|A_{n}\right|^{2}+\left|B_{n}\right|^{2}+\left|C_{n}\right|^{2}\right) .
\end{aligned}
$$

Applying Cauchy's inequality and Gallagher's lemma (see [1], Lemma 1) we find that for a fixed $n$,

$$
\begin{aligned}
& \text { (4.2) }\left|A_{n}\right|^{2} \\
& \leq P^{2} \max _{\substack{q \leq P \\
(a, q)=1}} \int_{-1 / Q}^{1 / Q}\left|D_{k}\left(\frac{a}{q}+\gamma\right)-\frac{C_{k}(q, a)}{\phi(q)} I_{k}(\gamma)\right|^{2} d \gamma \int_{0}^{1}\left|D_{1}(\gamma)\right|^{2} d \gamma \\
& \ll Q^{-2} y L^{2 B_{1}+2} \\
& \times \max _{\substack{q \leq P \\
(a, q)=1}} \int_{y / 2^{k}-Q / 2}^{\left(2^{k}+1\right) y / 2^{k}}\left|\sum_{\substack{t<m^{k} \leq t+Q / 2 \\
y / 2^{k}<m^{k} \leq\left(2^{k}+1\right) y / 2^{k}}}\left(\Lambda(m) e\left(\frac{a}{q} m^{k}\right)-\frac{C_{k}(a, q)}{\phi(q)}\right)\right|^{2} d t .
\end{aligned}
$$

Disregarding the powers of primes counted by $\Lambda(n)$ and introducing Dirichlet characters, we can derive from (4.2) that

$$
\begin{aligned}
\left|A_{n}\right|^{2} \ll & Q^{-2} y L^{3 B_{1}+2} \\
& \times \max _{q \leq P} \sum_{\chi \bmod q} \int_{y / 2^{k}-Q / 2}^{y / 2^{k}}\left|\sum_{y / 2^{k}<p^{k} \leq t+Q / 2}^{\prime}(\log p) \chi(p)\right|^{2} d t \\
& +Q^{-2} y L^{3 B_{1}+2} \\
& \times\left.\max _{q \leq P} \sum_{\chi \bmod q} \int_{y / 2^{k}} \sum_{y / 2^{k}<p^{k} \leq t+Q / 2}^{\prime}(\log p) \chi(p)\right|^{2} d t \\
& +\left.Q^{-2} y L^{3 B_{1}+2} \sum_{\left(2^{k}+1\right) y / 2^{k}} \int_{2^{k}-Q / 2}^{\prime} \sum_{y / 2^{k}<p^{k} \leq t+Q / 2}^{\prime}(\log p) \chi(p)\right|^{2} d t \\
& \times \max _{q \leq P} \sum_{\chi \bmod q\left(2^{k}+1\right) y / 2^{k}-Q / 2} \\
= & K_{1}+K_{2}+K_{3} .
\end{aligned}
$$

Estimating $K_{1}$ and $K_{3}$ trivially we obtain

$$
K_{1}+K_{3} \ll Q^{-2} y L^{4 B_{1}+2} Q\left(Q y^{1 / k-1} L\right)^{2}=Q y^{2 / k-1} L^{4 B_{1}+4} \leq y^{2 / k} L^{-A} .
$$

Substituting $t=v^{k}$ and taking into account that 


$$
Q v y^{-1} \ll Q v^{1-k} \ll \sqrt[k]{v^{k}+Q / 2}-\sqrt[k]{v^{k}} \ll Q v^{1-k} \ll Q v y^{-1},
$$

we apply the remark regarding Lemma 4.1 and find that

$$
K_{2} \ll Q^{-2} y L^{3 B_{1}+2} y^{1-1 / k} y^{3 / k}\left(\frac{Q}{y}\right)^{2} L^{-A-3 B_{1}-2}=y^{2 / k} L^{-A} .
$$

Summing up we get

$$
\left|A_{n}\right|^{2} \ll y^{2 / k} L^{-A} \text {. }
$$

For the estimation of $B_{n}$ we split the integral. If $|\gamma| \leq \gamma_{0}=y^{-1} L^{A+4 B_{1}+2}$ we have, by applying the Siegel-Walfisz theorem in short intervals (see (6) of $[6])$, the equality

$$
D_{1}\left(\frac{a}{q}\right)=\frac{\mu(q)}{\phi(q)} y+O_{E, \varepsilon, B_{1}}\left(y L^{-E}\right) .
$$

Thus by using partial summation and $I_{k}(\gamma) \ll y^{1 / k}$ we obtain, for a sufficiently large $E$,

$$
\int_{|\gamma| \leq \gamma_{0}}\left|D_{1}\left(\frac{a}{q}+\gamma\right)-\frac{\mu(q)}{\phi(q)} I_{1}(\gamma)\right|\left|I_{k}(\gamma)\right| d \gamma \ll y^{1 / k} L^{-A / 2-2 B_{1}} .
$$

If $\gamma_{0}<|\gamma| \leq 1 / Q$, we use Lemmas 4.2 and 4.8 of [11] to show $I_{k}(\gamma) \ll$ $1 / y^{(k-1) / k}|\gamma|$, and thus

$$
\begin{aligned}
\int_{\gamma_{0}<|\gamma| \leq 1 / Q} \ll & \left(\int_{0}^{1}\left(\left|D_{1}\left(\frac{a}{q}+\gamma\right)\right|^{2}+\left|I_{1}(\gamma)\right|^{2}\right) d \gamma\right)^{1 / 2} \\
& \times\left(\int_{\gamma_{0}<|\gamma| \leq 1 / Q}\left(|\gamma|^{-2} y^{2 / k-2}\right) d \gamma\right)^{1 / 2} \\
\ll & y^{1 / k} L^{-A / 2-2 B_{1}} .
\end{aligned}
$$

From (4.5) and (4.6) we can derive

$$
\left|B_{n}\right|^{2} \ll y^{2 / k} L^{-A} \text {. }
$$

We note that for $s \geq c k^{2} \log k$,

$$
\int_{0}^{1}\left|I_{k}(\gamma)\right|^{2 s} d \gamma \ll y^{2 s / k-1}
$$

(see Lemma in 5.2 of [12]). So together with $I_{1}(\gamma) \ll 1 /\|\gamma\|$ we have the following estimate for the integral in $C_{n}$

$$
\begin{aligned}
\int & \ll\left(\int_{0}^{1}\left|I_{k}(\gamma)\right|^{2 s} d \gamma\right)^{1 / 2 s}\left(\int_{1 / Q}^{1 / 2}|\gamma|^{-2 s /(2 s-1)} d \gamma\right)^{(2 s-1) / 2 s} \\
& \ll y^{1 / k-1 / 2 s} Q^{1 / 2 s}=y^{1 / k} L^{-B_{2} / 2 s},
\end{aligned}
$$


and so

$$
\left|C_{n}\right|^{2} \ll y^{2 / k} L^{-A} .
$$

From (4.1), (4.4), (4.7) and (4.8) we obtain

$$
\sum_{M} \ll H y^{2 / k} L^{-A},
$$

from which, together with (2.1) and (3.32), Theorem 2 follows.

5. Proof of Theorem 1. We need the following lemma:

Lemma 5.1. Let $T=[\sqrt[k]{H}]$, suppose $\eta$ is a small fixed number with $0<\eta<1 / 8, w$ is an arbitrarily large fixed number and $v=w+1-w \eta$. Set furthermore

$$
X=\left[(\log T) \frac{k-1 / 2}{20} \cdot \frac{\eta}{w v}\right] \quad \text { and } \quad S=\left[X^{v}\right] .
$$

Then for $x$ with $x^{1 / 3} \leq H \leq x$ and each fixed $D$ with $S \leq(\log H)^{D}$,

$$
\begin{gathered}
\sum_{x<n \leq x+H}\left|\sigma\left(n,(\log H)^{D}\right)-\prod_{p \leq S}\left(1+\frac{A(p, n)}{(p-1)^{2}}\right)\right|^{2} \\
\ll_{\varepsilon, k, w, \eta} H(\log T)^{-w+1+w \eta+\varepsilon .}
\end{gathered}
$$

Proof. The proof is literally the same as the one of Satz 1 of [10]. Furthermore, we know from Lemma 2.6 of [10] that

$$
\prod_{p \leq S}\left(1+\frac{A(p, n)}{(p-1)^{2}}\right) \gg L^{-1} .
$$

Using this we obtain

$$
\begin{aligned}
& E_{k}(x+H)-E_{k}(x) \\
& \ll y^{-2 / k} L^{2} \sum_{x<n \leq x+H}|R(n)-\sigma(n, P) P(n)|^{2} \\
& \quad+y^{-2 / k} L^{2} \sum_{x<n \leq x+H}\left|\sigma(n, P) P(n)-\prod_{p \leq S}\left(1+\frac{A(p, n)}{(p-1)^{2}}\right) P(n)\right|^{2} .
\end{aligned}
$$

Now Theorem 1 follows from Lemma 5.1 and Theorem 2 .

The author would like to thank Professor Dr. T. Zhan and Professor Dr. D. Wolke for their steady encouragement. 


\section{References}

[1] P. X. Gallagher, A large sieve density estimate near $\sigma=1$, Invent. Math. 11 (1970), 329-339.

[2] D. R. Heath-Brown, Prime numbers in short intervals and a generalized Vaughan's identity, Canad. J. Math. 34 (1982), 1365-1377.

[3] L. K. Hua, Some results in the additive prime number theory, Quart. J. Math. 9 (1938), 68-80.

[4] J. Y. Liu and T. Zhan, Estimation of exponential sums over primes in short intervals II, in: Analytic Number Theory, Proceedings of a Conference in Honor of Heini Halberstam, Vol. 2, Birkhäuser, 1996, 571-606.

[5] H. Mikawa, On the sum of a prime and a square, Tsukuba J. Math. 17 (1993), 299-310.

[6] A. Perelli and J. Pintz, On the exceptional set for Goldbach's problem in short intervals, J. London Math. Soc. (2) 47 (1993), 41-49.

[7] —, - Hardy-Littlewood numbers in short intervals, J. Number Theory 54 (1995), 297-308.

[8] A. Perelli and A. Zaccagnini, On the sum of a prime and a $k$-th power, Izv. Ross. Akad. Nauk Mat. 59 (1995), no. 1, 185-200.

[9] B. Saffari and R. C. Vaughan, On the fractional parts of $x / n$ and related sequences. II, Ann. Inst. Fourier (Grenoble) 27 (1977), no. 2, 1-30.

[10] W. Schwarz, Zur Darstellung von Zahlen durch Summen von Primzahlpotenzen. II, J. Reine Angew. Math. 206 (1961), 78-112.

[11] E. C. Titchmarsh, The Theory of the Riemann Zeta-Function, 2nd ed., Clarendon Press, Oxford, 1986.

[12] A. Zaccagnini, The exceptional set for the sum of a prime and a $k$-th power, Mathematika 39 (1992), 400-421.

[13] T. Zhan and J. Y. Liu, On a theorem of Hua, Arch. Math. (Basel) 69 (1997), $375-390$

Mathematisches Institut

Albert-Ludwigs-Universität Freiburg

Eckerstr. 1

79104 Freiburg, Germany 